(c) 2010 IEEE. Personal use of this material is permitted. Permission from IEEE must be obtained for all other uses, in any current or future media, including reprinting/republishing this material for advertising or promotional purposes, creating new collective works, for resale or redistribution to servers or lists, or reuse of any copyrighted component of this work in other works. 


\title{
A new LLMS Algorithm for Antenna Array Beamforming
}

\author{
Jalal Abdulsayed SRAR, Kah-Seng CHUNG and Ali MANSOUR
}

\author{
Dept. of Electrical and Computer Engineering, Curtin University of Technology, Perth, Australia \\ jalal.srar@google.com, k.chung@,curtin.edu.au, and a.mansour@,curtin.edu.au
}

\begin{abstract}
A new adaptive algorithm, called LLMS, which employs an array image factor, $A_{I}$, sandwiched in between two Least Mean Square (LMS) sections, is proposed for different applications of array beamforming. The convergence of LLMS algorithm is analyzed, in terms of mean square error, in the presence of Additive White Gaussian Noise (AWGN) for two different modes of operation; namely with either an external reference or self-referencing. Unlike earlier LMS based schemes, which make use of step size adaptation to enhance their performance, the proposed algorithm derives its overal error signal by feeding back the error signal from the second LMS stage to combine with that of the first LMS section. This results in LLMS being less sensitive to variations in input signal-to-noise ratio as well as the step sizes used. Computer simulation results show that the proposed LLMS algorithm is superior in convergence performance over the conventional LMS algorithm as well some of the more recent LMS based algorithms, such as constrained-stability LMS (CSLMS), and Modified Robust Variable Step Size LMS (MRVSS) algorithms. Also, the operation of LLMS remains stable even when its reference signal is corrupted by AWGN. It is also shown that LLMS performs well in the presence of Rayleigh fading.
\end{abstract}

Keywords-Adaptive array beamforming, LLMS algorithm, EVM, Rayleigh fading.

\section{INTRODUCTION}

In recent years, adaptive or smart antennas have become a key component for various wireless applications, such as radar, sonar, and $4 \mathrm{G}$ cellular mobile communications [1]. Its use leads to an increase in the detection range of radar and sonar systems, and the capacity of mobile radio communication systems. A summary of beamforming techniques is presented in [2].

Because of its simplicity and robustness, the LMS algorithm has become one of the most popular adaptive signal processing techniques adopted in many applications including antenna array beamforming. Over the past three decades, several improvements have been proposed to speed up the convergence of the LMS algorithm. These include variable-length LMS algorithm [3], Variable Step Size LMS (VSSLMS) algorithms [3-7], transform domain algorithms [8], and recently CSLMS [9] and MRVSS algorithms [10]. Because of their improved performance over other published LMS-based algorithms, both the CSLMS and MRVSS algorithms are included in this paper for performance comparison with the proposed LLMS scheme.

All the previously published LMS-based algorithms require an accurate reference signal for their proper operation. In some cases, several operating parameters are also required to be specified. As a result, the performance of such algorithm becomes highly dependent on the nature of the input signal [11].

This paper presents a very different approach to achieve fast convergence and low error floor with an LMS-based algorithm. The proposed LLMS algorithm involves the use of two LMS sections separated by an array image factor, $\boldsymbol{A}_{\boldsymbol{I}}$, as shown in Fig. 1. Such an arrangement maintains the low complexity generally associated with LMS. It can be shown that an N-element antenna array employing the LLMS algorithm involves $4 \mathrm{~N}+1$ complex multiplications and $2 \mathrm{~N}$ complex additions, i.e., slightly more than double the computational requirements of a conventional LMS scheme.

With the proposed LLMS scheme, the intermediate output, $y_{L M S 1}$, yielded from the first LMS section, $\left(\mathrm{LMS}_{1}\right)$, is multiplied by the image array factor $\left(\boldsymbol{A}_{\boldsymbol{I}}\right)$ of the desired signal. The resultant "filtered" signal is further processed by the second LMS section $\left(\mathrm{LMS}_{2}\right)$. For the adaptation process, the error signal of $\mathrm{LMS}_{2}, e_{2}$, is fed back to combine with that of $\mathrm{LMS}_{1}$, to form the overall error signal, $e_{L L M S}$, for updating the tap weights of $\mathrm{LMS}_{1}$. As shown in Fig. 1, a common external reference signal is used for both the two LMS sections, i.e., $d_{1}$ and $d_{2}$. This mode of operation, termed normal referencing, is analyzed in Section IIA. Moreover, the external reference signal may be replaced by $y_{L M S 1}$ in place of $d_{2}$, and $y_{L L M S}$ for $d_{1}$ to produce a selfreferenced version of the LLMS scheme, as described in Section II B.

The rest of the paper is organized as follows. In section II, the convergence of LLMS is analyzed in the presence of an external reference signal. This is then followed by an analysis involving the use of the estimated outputs, $y_{L M S 1}$ and $y_{L L M S}$ in place of the external reference. The latter is referred to as self-referencing from hereon. Results obtained from computer simulations for an eight element array are presented in Sections III. Finally, Section IV concludes the paper.

\section{CONVERGENCE OF THE PROPOSED LLMS Algorithm}

The convergence of the proposed LLMS algorithm has been analyzed with the following assumptions:

(i) The propagation environment is stationary.

(ii) The components of the signal vector $\boldsymbol{X}_{I}(j)$ should be independent identically distributed (iid). 


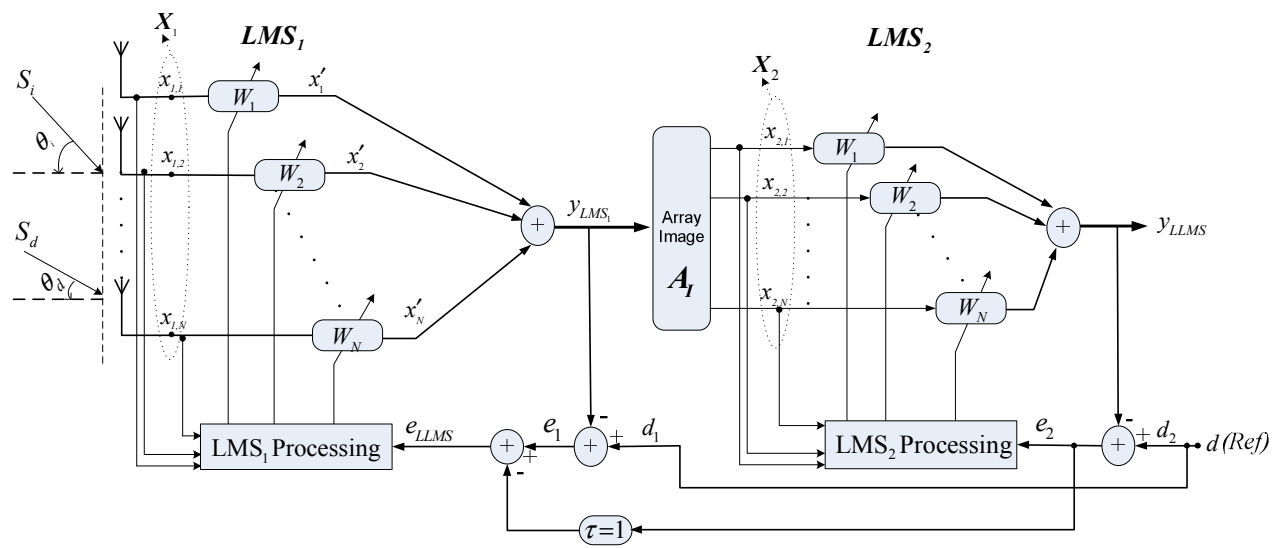

Figure1. The proposed LLMS algorithm with an external reference signal

(iii) All signals are zero mean and stationary at least to the second order.

A. Analysis with an external reference

From Fig. 1, the error signal for updating $\mathrm{LMS}_{1}$ at the $j^{\text {th }}$ iteration is given by

$$
\begin{array}{r}
e_{L L M S}(j)=e_{1}(j)-e_{2}(j-1) \\
\text { with } e_{i}(j)=d_{i}(j)-\boldsymbol{W}_{i}^{H}(j) \boldsymbol{X}_{i}(j)
\end{array}
$$

where $i=1$ for $\mathrm{LMS}_{1}$ and 2 for $\mathrm{LMS}_{2} ; \boldsymbol{X}_{i}(\cdot)$ and $\boldsymbol{W}_{i}(\cdot)$ represent the input signal and weight vectors, respectively of the $i^{\text {th }}$ LMS section..$(\cdot)^{H}$ denotes the Hermitian matrix of $(\cdot)$.

The input signal of $\mathrm{LMS}_{2}$ is derived from the $\mathrm{LMS}_{1}$, such that

$$
\boldsymbol{X}_{2}(j)=\boldsymbol{A}_{\boldsymbol{I}} y_{L M S 1}(j)=\boldsymbol{A}_{\boldsymbol{I}} \boldsymbol{W}_{1}^{H}(j) \boldsymbol{X}_{l}(j)
$$

The convergence performance of the LLMS algorithm can be analyzed in terms of the expected value of $e_{L L M S}^{2}$. It can be shown that:

$$
\begin{aligned}
\xi(j) \triangleq & E\left[\left|e_{L L M S}(j)\right|^{2}\right]=E\left[\left|e_{1}(j)-e_{2}(j-1)\right|^{2}\right] \\
= & E\left[|D(j)|^{2}\right]+\boldsymbol{W}_{1}^{H}(j) \boldsymbol{Q}(j) \boldsymbol{W}_{1}(j) \\
& -E\left[D(j) \boldsymbol{X}_{l}^{H}(j) \boldsymbol{W}_{1}(j)+D^{*}(j) \boldsymbol{W}_{1}^{H}(j) \boldsymbol{X}_{l}(j)\right]
\end{aligned}
$$

where $|\bullet|$ signifies modulus; $D(j)=d_{1}(j)-e_{2}(j-1)$, and $\boldsymbol{Q}$ is the correlation matrix of the input signals given by

$$
\boldsymbol{Q}(j)=E\left[\boldsymbol{X}_{l}(j) \boldsymbol{X}_{l}^{H}(j)\right]
$$

Consider the first term on the RHS of (2). With $d_{1}(j)$ and $e_{2}(j-1)$ being zero mean and uncorrelated based on the assumptions (ii) and (iii), this gives

$$
E\left[|D(j)|^{2}\right]=E\left[\left|d_{1}(j)\right|^{2}\right]+E\left[\left|e_{2}(j-1)\right|^{2}\right]
$$

Expanding (4) to include (1), and by assuming $d_{2}(j)=d_{1}(j)$, the first term on the RHS of (2) becomes

$$
\begin{aligned}
E[\mid & \left.\left.D(j)\right|^{2}\right]=E\left[\left|d_{1}(j)\right|^{2}\right]+E\left[\left|d_{2}(j-1)\right|^{2}\right] \\
& -\boldsymbol{W}_{L L M S}^{H}(j-1) \boldsymbol{Z}(j-1)-\boldsymbol{Z}^{H}(j-1) \boldsymbol{W}_{L L M S}(j-1) \\
& +\boldsymbol{W}_{\text {LLMS }}^{H}(j-1) \boldsymbol{Q}(j-1) \boldsymbol{W}_{\text {LLMS }}(j-1)
\end{aligned}
$$

where $\boldsymbol{W}_{L L M S}^{H}=\boldsymbol{W}_{2}^{H} \boldsymbol{A}_{\boldsymbol{I}} \boldsymbol{W}_{1}^{H}$, and $\boldsymbol{Z}(j)$ corresponds to the input signal cross-correlation vector given by

$$
\boldsymbol{Z}(j)=E\left[\boldsymbol{X}_{1}(j) d_{2}^{*}(j)\right]
$$

Let $d_{2}(j)=d_{1}(j)$, and by applying the assumptions (ii) and (iii), the last RHS term of (2) may be written as

$$
\begin{gathered}
E\left[D(j) \boldsymbol{X}_{l}^{H}(j) \boldsymbol{W}_{1}(j)+D^{*}(j) \boldsymbol{W}_{1}^{H}(j) \boldsymbol{X}_{l}(j)\right] \\
=\boldsymbol{Z}^{H}(j) \boldsymbol{W}_{1}(j)+\boldsymbol{W}_{1}^{H}(j) \boldsymbol{Z}(j)
\end{gathered}
$$

As a result, the mean square error $\xi$ as specified by (2) can be rewritten to include the results of (5) and (7) to become

$$
\begin{aligned}
\xi(j)= & E\left[\left|d_{1}(j)\right|^{2}\right]+E\left[\left|d_{2}(j-1)\right|^{2}\right] \\
& +\boldsymbol{W}_{L L M S}^{H}(j-1) \boldsymbol{Q}(j-1) \boldsymbol{W}_{L L M S}(j-1)-\boldsymbol{Z}^{H}(j) \boldsymbol{W}_{1}(j) \\
& -\boldsymbol{W}_{L L M S}^{H}(j-1) \boldsymbol{Z}(j-1)-\boldsymbol{Z}^{H}(j-1) \boldsymbol{W}_{L L M S}(j-1) \\
& -\boldsymbol{W}_{1}^{H}(j) \boldsymbol{Z}(j)+\boldsymbol{W}_{1}^{H}(j) \boldsymbol{Q}(j) \boldsymbol{W}_{1}(j)
\end{aligned}
$$

Differentiating (8) with respect to the weight vector $\boldsymbol{W}_{1}^{H}(j)$ then yields the gradient vector $\nabla(\xi)$ so that

$$
\nabla(\xi)=-\boldsymbol{Z}(j)+\boldsymbol{Q}(j) \boldsymbol{W}_{1}(j)
$$

By equating $\nabla(\xi)$ to zero, we obtain the optimal weight vector as

$$
\boldsymbol{W}_{\text {opt } 1}(j)=\boldsymbol{Q}^{-1}(j) \boldsymbol{Z}(j)
$$

when $\boldsymbol{X}_{l}$ is well excited and $\boldsymbol{Q}$ could be considered as full rank matrix.

This represents the Wiener-Hopf equation in matrix form. Therefore, the minimum MSE can be obtained from (10) and (8) to give

$$
\begin{aligned}
\xi_{\min } & =E\left[\left|d_{1}(j)\right|^{2}\right]+E\left[\left|d_{2}(j-1)\right|^{2}\right] \\
& -\boldsymbol{Z}^{H}(j) \boldsymbol{W}_{\text {opt } 1}(j)-\boldsymbol{Z}^{H}(j-1) \boldsymbol{W}_{\text {LLMS }}(j-1) \\
& +\boldsymbol{W}_{\text {LLMS }}^{H}(j-1) \boldsymbol{Z}(j-1)\left\{-1+\boldsymbol{A}_{\boldsymbol{I}}{ }^{H} \boldsymbol{W}_{2}(j-1)\right\}
\end{aligned}
$$


Based on (10) and (11), (8) becomes

$$
\xi=\xi_{\min }+\left(\boldsymbol{W}_{1}-\boldsymbol{W}_{o p t 1}\right)^{H} \boldsymbol{Q}\left(\boldsymbol{W}_{1}-\boldsymbol{W}_{o p t 1}\right)
$$

The error values of (12) are plotted as the theoretical curve in Fig. $2 b$.

Now, define

$$
\boldsymbol{V}_{1} \triangleq\left(\boldsymbol{W}_{1}-\boldsymbol{W}_{o p t 1}\right)
$$

so that (12) can be written as

$$
\xi=\xi_{\min }+\boldsymbol{V}_{l}^{H} \boldsymbol{Q} \boldsymbol{V}_{l}
$$

Differentiating (14) with respect to $\boldsymbol{V}_{I}^{H}$ will yield another form for the gradient, such that

$$
\nabla(\xi)=\boldsymbol{Q} \boldsymbol{V}_{1}
$$

Using eigenvalue decomposition (EVD) of $\boldsymbol{Q}$ in (15) yields

$$
\boldsymbol{Q}=\boldsymbol{q}_{l} \Lambda_{1} \boldsymbol{q}_{l}^{-1}=\boldsymbol{q}_{1} \Lambda_{1} \boldsymbol{q}_{I}^{H}
$$

where $\Lambda_{1}$ is the diagonal matrix of eigenvalues of $\boldsymbol{Q}$ for an $N$ element array, i.e.,

$$
\Lambda_{1}=\operatorname{diag}\left[E_{1}, E_{2}, \cdots, E_{N}\right]
$$

where $\operatorname{diag}[\ldots]$ is the diagonal of $\boldsymbol{Q}$.

For steepest descent algorithms, the weight vector is updated according to

$$
\boldsymbol{W}_{1}(j+1)=\boldsymbol{W}_{1}(j)-\mu_{1} \nabla \xi(j)
$$

where $\mu_{1}$ is the convergence constant that controls the stability and the rate of adaptation of the weight vector, and $\nabla \xi(j)$ is the gradient at the $j^{\text {th }}$ iteration.

We may rewrite (18) in the form of a linear homogeneous vector difference equation using (13), (15) and (16) to give

$$
\boldsymbol{V}_{1}(j+1)=\boldsymbol{V}_{1}(j)-\mu_{1} \boldsymbol{Q}_{1} \boldsymbol{V}_{1}(j)
$$

Alternatively, (19) can be written as

$$
\boldsymbol{V}_{1}(j)=\boldsymbol{q}_{l}\left(\boldsymbol{I}-\mu_{1} \Lambda_{1}\right)^{j} \boldsymbol{q}_{l}^{H} \boldsymbol{V}_{1}(0)
$$

By substituting (20) in (14), the MSE at the $j^{\text {th }}$ iteration is given by

$$
\xi(j)=\xi_{\min }+\boldsymbol{V}_{1}^{H}(0) \boldsymbol{q}_{1}\left|\left(\boldsymbol{I}-\mu_{1} \boldsymbol{\Lambda}_{1}\right)^{j}\right|^{2} \boldsymbol{q}_{1}^{H} \boldsymbol{V}_{1}(0)
$$

From (21), the asymptotic value of $\xi$ becomes

$$
\lim _{j \rightarrow \infty} \xi(j)=\xi_{\min }
$$

\section{B. Analysis of the self-referencing scheme}

Next, consider the case when the external reference is being replaced by internally generated signals, such that

$$
d_{1}(j)=y_{L L M S}(j-1), \text { and } d_{2}(j)=y_{L M S 1}(j)
$$

As a result of these changes, and note that the error signal $e_{2}=d_{2}-y_{\text {LLMS }}$, we can redefine $D(j)$ in (2) as

$$
D(j)=2 y_{L L M S}(j-1)-y_{L M S 1}(j-1)
$$

Based on the definition of (24), we reanalyze the MSE as defined in (2) to yield

$$
\begin{aligned}
\boldsymbol{\xi}(j)= & E\left[|D(j)|^{2}\right]-\boldsymbol{Z}^{\prime H}(j) \boldsymbol{W}_{1}(j) \\
& -\boldsymbol{W}_{1}^{H}(j) \boldsymbol{Z}^{\prime}(j)+\boldsymbol{W}_{1}^{H}(j) \boldsymbol{Q}(j) \boldsymbol{W}_{1}(j)
\end{aligned}
$$

where $\boldsymbol{Z}^{\prime}(j)$ corresponds to the input signal crosscorrelation vector given by

$$
\boldsymbol{Z}^{\prime}(j)=E\left[\boldsymbol{X}_{l}(j) D^{*}(j)\right]
$$

The error values obtained from (25) are plotted as the theoretical curve in Fig. 3.

By following the same analyzing steps of (12) to (22), it can be shown that the proposed LLMS algorithm will converge under the condition of self-referencing.

\section{Simulation RESULtS}

The performance of LLMS algorithm has been studied by means of MATLAB simulation. For comparison purposes, results obtained with the conventional LMS, CSLMS and MRVSS algorithms are also presented. For the simulations, the following parameters are used:

- A linear array consisting of 8 isotropic elements.

- A Binary Phase Shift Keying (BPSK) signal arriving at an angle of $0^{\circ}$, or if specified at $-20^{\circ}$.

- An AWGN channel with a BPSK interference signal arriving at $\theta_{i}=45^{\circ}$ having the same amplitude as the desired signal unless otherwise stated.

- All weight vectors are initially set to zero.

- For the Rayleigh fading case, $f_{d}=60 \mathrm{~Hz}$. The input signal at each array element undergoes independent Rayleigh fading.

Table 1 tabulates the values of the various constants adopted by the simulations for the four different adaptive algorithms. The constants for MRVSS are taken from [5].

For a digital modulated signal, it is also convenient to make use of the Error Vector Magnitude (EVM) as an accurate measure of any distortion introduced by the adaptive scheme on the received signal at a given signal-tonoise ratio $(S N R)$. EVM is defined as [12]

$$
E V M_{R M S}=\sqrt{\frac{1}{K P_{o}} \sum_{j=1}^{K}\left|S_{r}(j)-S_{t}(j)\right|^{2}}
$$

where $K$ is the number of symbols used, $S_{r}(j)$ is the normalized $j$ th output of the beamformer, and $S_{t}(j)$ is the $j$ th transmit symbol. $P_{o}$ is the normalized transmit symbol power.

TABLE I. VAlues of The Constants Uesd in Simulation

\begin{tabular}{|c|c|}
\hline Algorithm & Normal Channel \\
\hline LMS & $\mu=0.05$ \\
LLMS & $\mu_{1}=\mu_{2}=0.05$ \\
CSLMS & $\varepsilon=0.05$ \\
MRVSS & $\begin{array}{l}\beta_{\max }=1, \beta_{\min }=0, v=5 e-4, \mu_{\max }=0.2, \mu_{\min }=1 e-4 \\
\mu=\mu_{\max }, \alpha=0.97, \gamma=4.8 e-4, \eta=0.97\end{array}$ \\
\hline
\end{tabular}




\section{A. Performance with an external reference}

First, the performances of the LLMS, CSLMS, MRVSS and LMS schemes have been studied in the presence of an external reference signal. The convergence performances of these schemes are compared based on the ensemble average squared error $\left(\tilde{e}^{2}\right)$ obtained from 100 individual simulation runs. The results obtained for different values of input $S N R$, and step size, $\mu_{1}$ and $\mu_{2}$, are presented.

Figs. $2 \mathrm{a}-2 \mathrm{c}$ show the convergence behaviors of the four adaptive schemes for SNR values of 5, 10, and $15 \mathrm{~dB}$, respectively. For the proposed LLMS scheme, the theoretical convergence error calculated using (12) for $\mathrm{SNR}=10 \mathrm{~dB}$ is also shown in Fig. 2b. It verifies a close agreement between the simulated and theoretical error plots for LLMS. Also, it is observed that under the given conditions, LLMS algorithm converges much faster than the other three schemes. Furthermore, the error floor of LLMS is less sensitive to changes in input SNR.

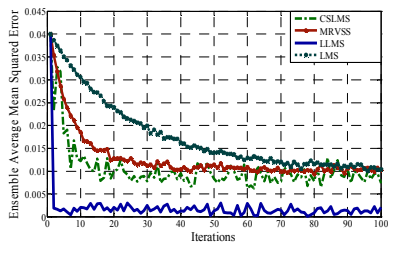

(a) $S N R=5 \mathrm{~dB}$

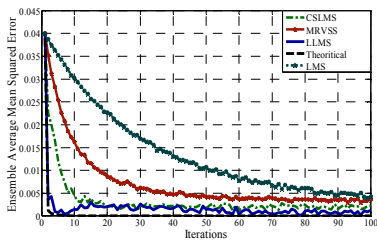

(b) $S N R=10 \mathrm{~dB}$

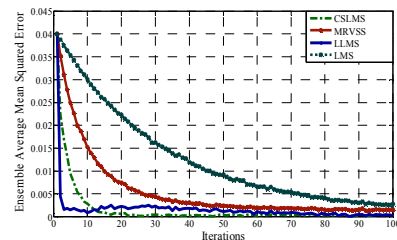

(c) $S N R=15 \mathrm{~dB}$

Fig. 2. The convergence of LLMS, CSLMS, MRVSS and LMS using the parameters given in Table I, for three different values of input $S N R$.

\section{B. Performance with self-referencing}

As shown in Fig. 2, the LLMS algorithm converges rapidly within ten iterations. Once this occurs, the intermediate output, $y_{L M S 1}$, tends to resemble the desired signal $s_{d}(t)$, and may then be used in place of the external reference $d_{2}$ for the current iteration of the $\mathrm{LMS}_{2}$ section. As the $\mathrm{LMS}_{2}$ section converges, its output $y_{\text {LLMS }}$ becomes the estimated $s_{d}(t)$. As a result, $y_{L L M S}$ may be used to replace $d_{1}$ as the reference for the $\mathrm{LMS}_{1}$ section. This feedforward and feedback arrangement enables the provision of selfreferencing in LLMS, and allows the external reference signal to be discontinued after an initial four iterations.

The ability of the LLMS algorithm to maintain operation with the internally generated reference signals is demonstrated in Fig. 3. It shows that the LMS, CSLMS, MRVSS algorithms are unable to converge without the use of an external reference signal. For comparison, the theoretical convergence error is also plotted in Fig. 3.

\section{Performance with a noisy reference signal}

The performances of LLMS, CSLMS, MRVSS and LMS have also been investigated when the reference signal used is corrupted by AWGN. Fig. 4 shows the ensemble average of the mean square error, $\bar{\xi}$, obtained from 100 individual simulation runs, as a function of the ratio of the rms noise level $\sigma$ to the amplitude of the reference signal $S_{R}$.

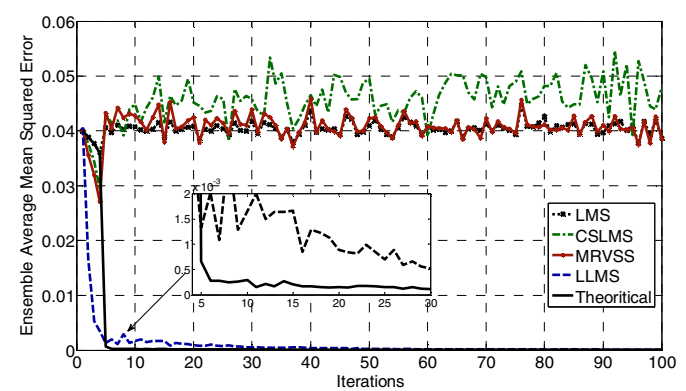

Fig. 3. The convergence of LLMS with self-referencing using the parameters given in Table I, for $S N R=10 d B$. An external reference is used for the initial four iterations.

It is interesting to note that the LLMS algorithm is very tolerant to noisy reference signal. On the other hand, the LMS, CSLMS and MRVSS algorithms are quite sensitive to the presence of noise in the reference signal. As shown in Fig. 4, the values of $\bar{\xi}$ associated with LLMS, remains very small even when the rms noise level becomes as large as the reference signal.

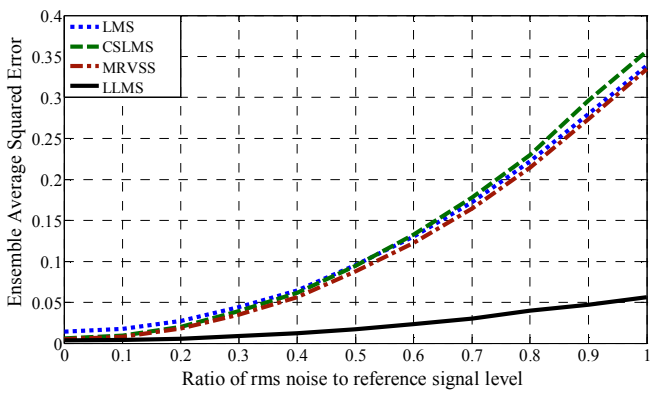

Fig. 4. The influence of noise in the reference signal on the mean square error $\xi$ for an input $S N R$ is $10 \mathrm{~dB}$. The parameter values used are as given in Table I.

Next, the beam patterns obtained with the four adaptive algorithms for three different values of rms noise-toreference signal ratio $\left(\sigma / S_{R}\right)$ are shown in Figs. 5a-c. In this case, the input SNR is $10 \mathrm{~dB}$ and the angle of arrival of the desired signal is $-20^{\circ}$. Figs. $5 \mathrm{a}$ and $\mathrm{b}$, show that the resultant beam patterns of LLMS remain almost the same as that achieved with a noise free reference (Fig. 5c). On the contrary, the gains of the other three beamformers are reduced when their reference signals are corrupted by AWGN.

\section{LLMS operating in a flat fading channel}

The influence of Rayleigh fading on the performances of the four adaptive beamforming schemes has been investigated under the following conditions:

- The desired signal arrives at an angle of $0^{\circ}$.

- Two interfering signals with the same amplitude as the desired signal arrive at $-30^{\circ}$ and $45^{\circ}$, respectively. 
- The input signal at each antenna element undergoes independent Rayleigh fading.

- Each simulation run lasts for $16 \mathrm{M}$ samples.

Figs. 6 a and b, show the resultant EVM values obtained using the four different algorithms. It is observed that LLMS

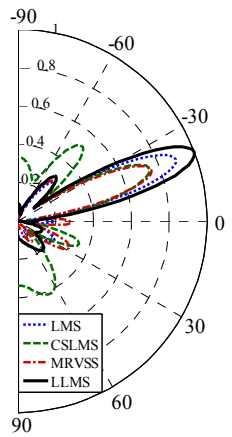

(a) $\sigma_{N} / S_{R}=1 / \sqrt{2}$

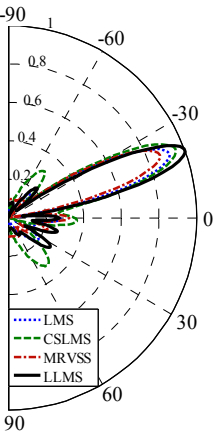

(b) $\sigma_{N} / S_{R}=1 / 2 \sqrt{2}$ (c) $\sigma_{N} / S_{R}=0$

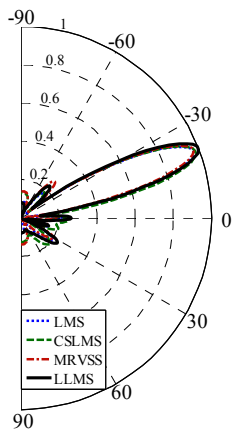

Fig. 5. The beams patterns achieved with the LLMS, CSLMS, MRVSS and LMS algorithms when the reference is corrupted by AWGN. The angle of arrival of the desired signal is $-20^{\circ}$.

can achieve lower EVM values, which indicate better signal fidelity, particularly at lower input SNR values. This suggests that LLMS is better able to handle time varying signals compared with conventional LMS, CSLMS and MRVSS.

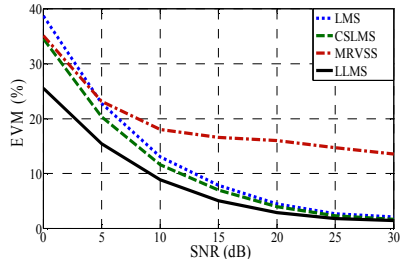

(a) Fading with Interference

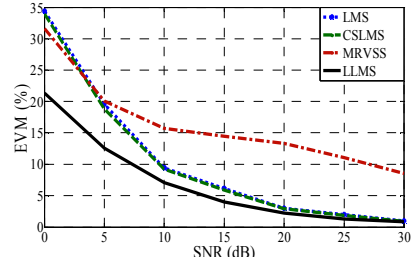

(b) Fading without Interference
Fig. 6. The EVM values obtained with the LLMS, CSLMS, MRVSS and LMS algorithms for different input SNR under Rayleigh fading channel.

\section{CONCLUSIONS}

A new adaptive algorithm for beamforming, called LLMS, is presented and analyzed. The convergence of LLMS scheme has been analyzed assuming the use of an external reference signal. This is then extended to cover the case that makes use of self-referencing. The convergence behaviors of the LLMS algorithm with different step size combinations of $\mu_{1}$ and $\mu_{2}$ have been demonstrated by means of Matlab simulations under different input SNR conditions.

It is shown that the proposed LLMS algorithm can achieve rapid convergence, typically within a few iterations. Furthermore, the steady state MSE of LLMS is quite insensitive to input SNR. Unlike the conventional LMS, CSLMS and MRVSS algorithms, the proposed LLMS scheme is able to operate with noisy reference signal. Once the initial convergence is achieved, within a few iterations, the LLMS scheme can maintain its operation through selfreferencing. In addition, it is shown that the use of LLMS can maintain the fidelity of the signal in the presence of Rayleigh fading, as indicated by the resultant low EVM values. Overall, LLMS performs better than conventional LMS, CSLMS and MRVSS algorithms under the various operating conditions considered in this paper. This has been achieved with a complexity slightly more than twice that of a conventional LMS scheme.

\section{REFERENCES}

1. Martin-Sacristan, D., et al., "On the Way Toward ForthGeneration Mobile: 3GPP LTE-Advanced", EURASIP Journal on Wireless Communications and Networking, vol. 2009, Jun. 2009.

2. Stine, J.A., "Exploiting smart antennas in wireless mesh networks using contention access", IEEE Trans. on Wireless Communications, vol. 13(2), pp. 38-49, 2006.

3. Nascimento, V.H., "Improving the initial convergence of adaptive filters: variable-length LMS algorithms", 14th International Conference on Digital Signal Processing, Santorini, Greece, pp. 667-670, July 2002.

4. Zhao, S., Z. Man, and S. Khoo, "A Fast Variable StepSize LMS Algorithm with System Identification", 2nd IEEE Conference on Industrial Electronics and Applications, Harbin, China, pp. 2340-2345, May 2007.

5. Kwong, R.H. and E.W. Johnston, "A variable step size LMS algorithm", IEEE Trans. on Signal Processing, vol. 40(7), pp. 1633-1642, 1992.

6. Aboulnasr, T. and K. Mayyas, "A robust variable stepsize LMS-type algorithm: analysis and simulations", IEEE Trans. on Signal Processing, vol. 45(3), pp. 631639, 1997.

7. Wee-Peng, A. and B. Farhang-Boroujeny, "A new class of gradient adaptive step-size LMS algorithms", IEEE Trans. on Signal Processing, vol. 49(4), pp. 805-810, 2001.

8. Lobato, E.M., O.J. Tobias, and R. Seara, "Stochastic modeling of the transform-domain ELMS algorithm for correlated Gaussian data", IEEE Trans. on Signal Processing, vol. 56(5), pp. 1840-1852, 2008.

9. Górriz, J.M., et al., "Speech enhancement in discontinuous transmission systems using the constrained-stability least-mean-squares algorithm", Acoustical Society of America, vol. 124(6), pp. 36693683, Dec. 2008.

10.Zou, k. and X. Zhao. "A new modified robust variable step size LMS algorithm", 4th IEEE Conference on Industrial Electronics and Applications, Xi'an, China, May 2009.

11. Mathews, V.J. and Z. Xie, "A stochastic gradient adaptive filter with gradient adaptive step size", IEEE Trans. on Signal Processing, vol. 41(6), pp. 2075-2087, 1993.

12. Arslan, H. and H. Mahmoud, "Error vector magnitude to SNR conversion for nondata-aided receivers", IEEE Trans. on Wireless Communications, vol. 8(5), pp. 26942704, 2009. 